\title{
EVOLUTION OF INTEGRATED INERTIAL MEMS TECHNOLOGY
}

\author{
Michael W. Judy \\ Analog Devices, Inc. \\ Micromachined Products Division \\ Cambridge, MA 02139-3556
}

\begin{abstract}
Integrated inertial surface-micromachined MEMS technology is presented as an example of how accelerometers and angular-rate sensors are evolving in response to competition, innovation and customer requirements. These market forces are commoditizing the products and impacting key technologies such as fabrication, assembly, packaging, test, and design. Current and future directions of integrated inertial MEMS technology will be disclosed, as well as how these advances will enable both future inertial and non-inertial MEMS products.
\end{abstract}

\section{BACKGROUND}

Micromachined inertial products have been manufactured in volume since the mid 1980's, although initially in small volumes and for characterization and instrumentation applications [1,2]. These non-integrated accelerometers and gyros were bulkmicromachined sensors using predominately piezoresistance to measure the displacement resulting from an applied acceleration or angular rate. The limited application of MEMS inertial sensors was due to their high cost relative to other simpler devices. Lowperformance applications, such as measuring tilt or bump detection, were adequately met by mercury switches and pendulums. When the US government mandated that all cars must have airbag safety systems, it created what Peterson called a "starved market segment" [3]. This market required a highvolume ( $>10^{7}$ units/year), low-cost, high-reliability, high-quality, and high-performance accelerometer. Although the bulk micromachined sensors met the performance specifications, they were lacking on the other criteria, especially a path to low cost.

Polysilicon surface micromachining, which had been developed during the early 1980's, offered an attractive alternative that sacrificed some performance specifications for lower costs and higher quality. The reduced performance was due to the much smaller inertial masses that are created using surface micromachining. A typical bulk micromachined sensor mass is approximately $1 \mathrm{mg}$ while a surface micromachined sensor mass is $1 \mu \mathrm{g}$, or 1000 times lower. The lower mass translates into more Brownian noise, higher resonant frequencies and lower spring constants or restoring forces. Surface micromachining; however, promised much lower costs through smaller sensors and resulting die sizes, more compatibility with standard IC fabrication and assembly techniques, and reduced integration complexity between circuits and MEMS.

Recent competition to access the rapidly growing market for inertial sensors in cars, in particular the burgeoning low-g market, has been quite acute. There are more than a dozen companies with over 100 products to address these markets. Two broad classes of MEMS integration strategies have been developed: one-chip and two-chip solutions - otherwise known as integrated and unintegrated. The technology followed by most companies (e.g., Bosch, Motorola, STMicro, Infineon, Kionix, etc.) is the two-chip solution where a MEMS-only chip and a companion electronics chip are combined in a single package. The primary advantage of this approach is that the MEMS and circuit processing do not interact, thus allowing each process and design to be optimized independently and quickly. This approach allows the company to focus its resources on the value added parts of the product: the circuit and MEMS design and the MEMS process. The other advantage of this approach is that the total processing cycle time is less. The disadvantage is that a considerably larger sensor is required to compensate for the additional parasitic capacitances. This makes it very difficult to migrate to smaller less expensive packages. The one-chip solution, or integrated solution, has the advantage of a smaller MEMS structure and thus resulting die size. This provides access to much smaller packages and thus a path to lower costs. The down side is that this process has more complexity, predominately due to circuit-MEMS interactions, and has longer fabrication time and thus longer product development time. Neither approach, integrated or unintegrated, has dominated the market, nor are they likely to do so in the near future.

The application of accelerometers and gyros in cars over the last decade has multiplied from one accelerometer in the center module to more than a dozen accelerometers and gyros participating in not only passive but also active safety systems. The passive safety systems include all of the airbag systems, seatbelt tensioners and rollover protection. In an active safety system, like vehicle dynamic control (VDC), certain systems within the automobile are temporarily under the control of the VDC system, not the driver. As a result of this relinquishment of control, the criteria for MEMS quality and reliability have become even more stringent. The low-g market has been much more dynamic with excitement about a given application followed by a moderation as the real demand for the application did not materialize into highvolume demand. An example of this type of market is using inertial inputs for computer game controllers (e.g., Nintendo Gameboy). The emerging low-g markets are in laptops, cell phones, and PDAs. In these new markets the sensors are used for all manner of bump/shock sensing and tilt sensing such as menu scrolling and selecting.

\section{TECHNOLOGY EVOLUTION THEMES}

High-volume inertial sensors, as with all markets, have been subject to the following pressures through competition and the generation of new market opportunities:

1. Lower cost

2. Smaller size and weight

3. Higher quality \& reliability

4. Higher performance

5. Total ownership cost

The first, and many would argue the second and third priority, is reducing the price of the MEMS component. For all high volume markets, price is the governing criteria. If the component suppliers 
are unable to meet the price expectations, the system manufacturer will likely either stick with an existing solution that may have worse performance or delay the entire program. Depending on the market there are different levels of quality and reliability requirements. Automotive suppliers, for example, demand $<1 \mathrm{ppm}$ failures in the field, while consumer electronic equipment suppliers have less stringent requirements.

In response to the market pressures discussed above three different technology development themes have been followed by every competitor:

1. Higher levels of efficiency (e.g., die size)

2. Increasing use of standard IC equipment (e.g., foundries)

3. Product differentiation (e.g., performance \& support)

Increasing efficiency affects every aspect of design and manufacturing. All competitors have worked hard to produce smaller structures and packages with more functionality and higher performance. This evolution puts ever more emphasis on collaboration between all aspects of design and manufacturing, to resolve both yield and quality issues, but also to design the nextgeneration sensor with improved performance. The interdisciplinary nature of MEMS demands this intimate collaboration. The special processing-equipment requirement for MEMS devices means that additional fixed expenses must be incurred beyond those typical for any microelectronics facility. Improving the technology to remove these requirements provides access to lower cost foundry and assembly facilities; however, it also creates dependence as well. The final step of product differentiation usually focuses on some aspect of the product, such as package size or a performance specification. It is also vital to provide an adequate customer-support organization to help customers with implementation of the components in both their production lines as well as their intended applications. This goes beyond conventional design-in assistance and extends into system (i.e., mechanical design) and manufacturing process development.

\section{FRONT-END INTEGRATED MEMS PROCESS TECHNOLOGY}

A current high-volume integrated inertial MEMS process technology is the Analog Devices process called $i$ MEMS ${ }^{\circledR}$. This process has been developed over the past 15 years and is a robust $3-\mu \mathrm{m}$ BiCMOS process integrated with a surface-micromachined polysilicon MEMS device (Figure 1) [4]. Other important characteristics of the process include: laser trimmed thin-film resistors and both $5-\mathrm{V}$ and $24-\mathrm{V}$ devices. Over the last several years at ADI a next-generation integrated MEMS technology named SOIMEMS has also been developed and will also be discussed.

There is another integrated technology that is currently being commercialized at MEMSIC, where a thermal accelerometer is integrated with foundry CMOS. This process incorporates a low fixed cost manufacturing process with an X-Y accelerometer that is not susceptible to several failure mechanisms intrinsic to capacitive based sensors. The performance of these devices appears to be limited, but time will tell if the emerging applications will be able to use these devices. If so, this approach will be very effective within its niche.
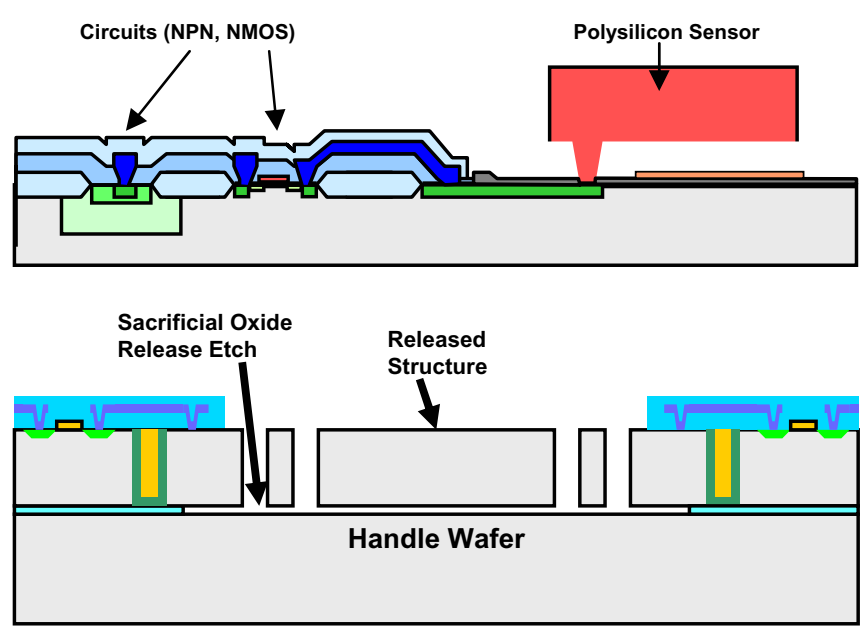

Figure 1: a) iMEMS: 3- $\mu \mathrm{m}$ BiCMOS interleaved with 2-4 $\mu \mathrm{m}$ polysilicon. b) SOIMEMS: 0.6- $\mu \mathrm{m}$ CMOS integrated with 10- $\mu \mathrm{m}$ SOI MEMS devices.

iMEMS: The circuit-processing and MEMS-processing steps are interweaved in $i$ MEMS. The process begins with the circuit process up to gate planarization, and then continue with all MEMS processing prior to release, then finishing the circuit process (i.e., metallization and passivation), and finally removing the sacrificial oxide. Several different versions of the core $i$ MEMS technology have been used in production. Each version improved on the key metrics of performance, yield, reliability, and area utilization. The primary feature is the migration to thicker polysilicon for the device layer. Increasing thickness, or rather increasing the aspect ratio of the resulting capacitor gap, increases the sensitivity of the differential capacitors, provides a stiffer suspension and produces a more heavily damped sensor. The original polysilicon thickness was $2 \mu \mathrm{m}$ and has migrated to $4 \mu \mathrm{m}$. In addition, the number of interconnect layers under the released structure were increased to two, which facilitated complex designs such as the ADXRS150 gyro. Finally, the sacrificial oxide thickness was increased to reduce the parasitic capacitance of the structural layer, which impacts noise performance.

Table 1: Details of the different versions of the $i$ MEMS process. *Version 3 has reduced parasitics for the MEMS interconnect layers.

\begin{tabular}{|c|c|c|c|c|}
\hline $\begin{array}{c}i \text { MEMS } \\
\text { Version }\end{array}$ & $\begin{array}{c}\text { Ground } \\
\text { Layer }\end{array}$ & $\begin{array}{c}\text { Interconnect } \\
\text { Layers }\end{array}$ & $\begin{array}{c}\text { Sacrificial } \\
\text { Oxide }\end{array}$ & $\begin{array}{c}\text { Poly Si } \\
\text { Thickness }\end{array}$ \\
\hline 0 & Bare Si & 1 & $1.6 \mu \mathrm{m}$ & $2 \mu \mathrm{m}$ \\
\hline 1 & Poly Si & 2 & $1.6 \mu \mathrm{m}$ & $3 \mu \mathrm{m}$ \\
\hline 2 & Poly Si & 2 & $2.0 \mu \mathrm{m}$ & $4 \mu \mathrm{m}$ \\
\hline $3^{*}$ & Poly Si & 2 & $2.0 \mu \mathrm{m}$ & $4 \mu \mathrm{m}$ \\
\hline
\end{tabular}

SOIMEMS: To meet the demands of higher efficiency, higher functionality and more robust structures, ADI has been developing a next-generation process over the last several years. The result is a $0.6-\mu \mathrm{m}$ BiCMOS double-metal process with postpackage trim capability. The process integrates MEMS and electronics using a $10-\mu \mathrm{m}$-thick SOI layer for both the electronics and MEMS structures (Figure 1). The MEMS structures are isolated from the surrounding SOI by trench isolation. The integration strategy is similar to $i$ MEMS in that there is MEMS processing at the beginning (trench isolation) and the end of the entire process (structure etch and release), but has the advantage that all of the circuit processing is done in one process module. 
This process module is run in a circuit foundry instead of a custom fabrication facility, thus reducing fabrication cost.

The primary challenge for this approach has been to engineer the pre-processed wafers, such that there is a minimal impact on the foundry process module. The complexity of this task should not be trivialized and the adoption of a finer geometry CMOS process would be met with similar development challenges and times.

Release \& Surface treatment: Both $i$ MEMS and SOIMEMS use a release process where temporary photoresist pedestals prevent the structures from collapsing during drying. Vapor HF is a viable alternative that is used by many of the MEMS-only competitors that removes the requirement for photoresist pedestals. An alternative protection layer, such as polyimide, is required since photoresist is permeable to vapor HF.

An effective surface treatment is also required to insure that the MEMS devices do not stick when brought into contact during subsequent handling. In the original anti-stiction coating process at ADI, the coating agent was dispensed into the ceramic package just prior to lid seal. Several years ago a wafer-level anti-stiction coating process was put into production. This improved the uniformity of the coating as well as removed the requirement for a special processing step in assembly.

\section{BACK-END PROCESS TECHNOLOGY: ASSEMBLY, PACKAGING \& TEST}

The main items of interest in back-end processing are how to prevent the released MEMS device from being damaged or contaminated during the assembly process and to protect the device from the environment during its intended and unintended use.

Dicing: The initial assembly flow starts with dicing the completed wafers. To protect the released MEMS devices from the slurry during dicing, a temporary cap is created from two layers of tape suspended on a film frame [6]. One layer of tape is uniform and one has holes punched though it to create a cavity that will cover the MEMS device during dicing.

Wafer Capping: While the above dicing process works well, the released MEMS devices are still exposed to the environment of the assembly facility during the remainder of the assembly flow. This requires working with the assembly contractor to improve cleanliness and to institute process monitoring and inspections to insure that contamination is minimized to an acceptable level. Since the current failure rate in cars of the airbag accelerometers built with this process is $<1 \mathrm{ppm}$ the goal has been achieved. However, this assembly flow is custom and thus costs a premium when compared with open-market assembly of integrated circuits. This cost driver has motivated the development of a wafer-capping approach (Figure 2). The cap is fabricated with a combination of deep silicon etching and wafer sawing and is bonded to the MEMS die directly over the circuitry using a screen printed glass-frit seal [7]. These techniques provided a cap with tight design rules that protect the structure during the assembly flow as well as establish improved control over the environment surrounding the release structure. The capped wafer can then be sent to any assembly contractor, which will significantly reduce cost.

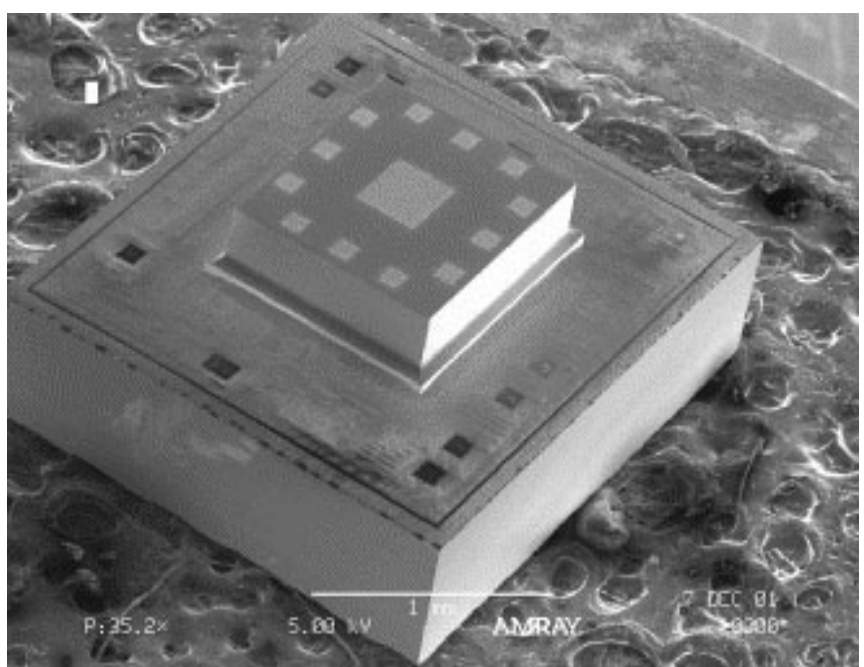

Figure 2: SEM image of a capped iMEMS® accelerometer.

Package evolution: MEMS packaging has traditionally been the most expensive part of the manufacturing process so there is great incentive in adopting lower cost packaging solutions. ADI is no exception to this industry wide trend. Figure 3 shows the evolution of the accelerometer packages at ADI with each package shown at the same scale. At each stage of development a standard IC package was adopted for integration with the MEMS devices. For the first 3 packages, hermetic cavities were created to house the released MEMS dies. With the development of the capping technology described above, low cost plastic packaging has become accessible. The most prevalent plastic package is the Lead Frame Chip Scale Package (LFCSP); commonly known by the trademarked assembly contractor names of MLF, QFN and MLP. This leadless package produces a smaller and lighter package than an equivalent leaded package such as an SOIC. The LFCSP package is also desirable from a manufacturing point of view as it uses a very similar process to leaded transfer molded packages, thus reducing capital investments.
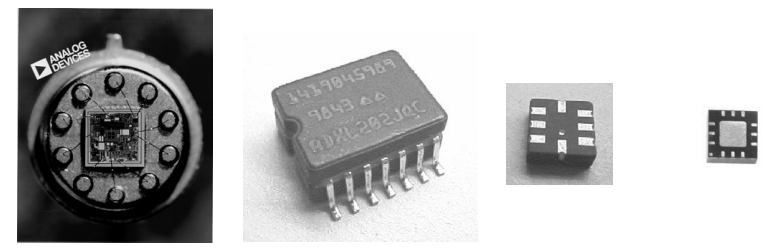

\begin{tabular}{|c|c|c|c|c|}
\hline & Header & Cerdip/Pak & LCC & LFCSP \\
\hline $\mathrm{X}_{\mathrm{mm}}$ & 10 & 10 & 5 & 4 \\
\hline$Y_{\mathrm{mm}}$ & 10 & 10 & 5 & 4 \\
\hline$Z_{\mathrm{mm}}$ & 7 & 5 & 3 & $1.5 \rightarrow 0.9$ \\
\hline
\end{tabular}

Figure 3: The package development of ADI's accelerometer product line: TO-100, CERPAK, LCC, LFCSP. The packages are shown at the same scale.

Test: In high-volume markets, automated testers are used to keep costs at a minimum. Figure 4 shows a typical IC tester and handler combined with a shaker which applies the appropriate input acceleration or angular rate. Although the tester-handler is standard equipment, the incorporation of a shaker means that the resulting platform is custom equipment and thus more expensive than on the open market. In addition to the typical electrical tests, the following sensor specifications must be measured; sensitivity, 


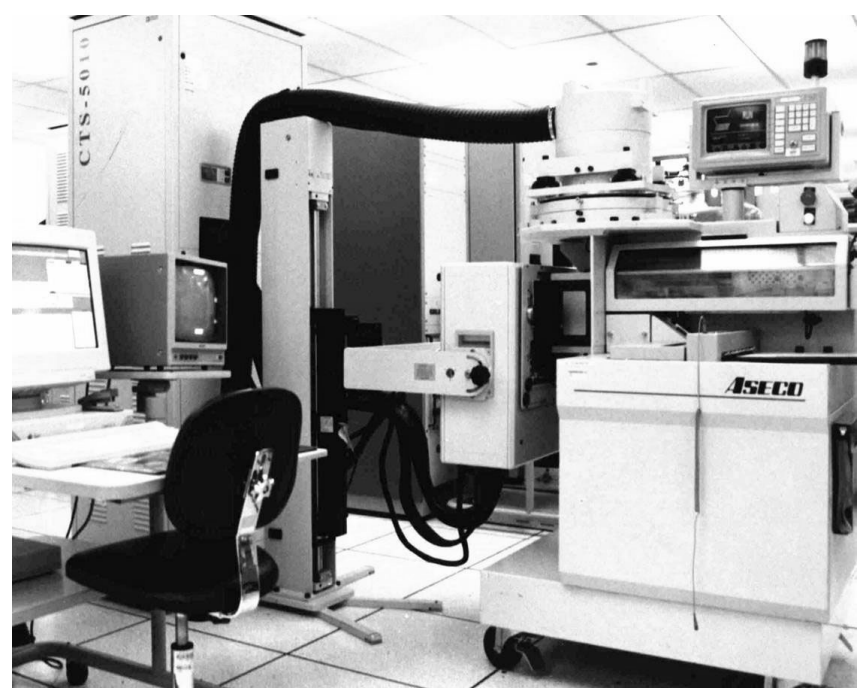

Figure 4: Automatic tester with integrated handler.

offset, self-test, and noise. Insuring sensitivity is measured to within $\pm 1 \%$ is a difficult challenge that is accomplished by using calibration standards that are measured at the same time as the device under test. Noise is a critical performance parameter, and great care must be taken to insure that electrical as well as mechanical noise and vibration do not interfere with this measurement. This has become an even greater challenge as the throughput rates of the testers are pushed faster. The development of multi-axis accelerometers and gyroscopes have also pushed the test technology.

\section{DESIGN}

Figure 5 shows the product evolution of the airbagaccelerometer family at ADI. The most obvious characteristic is the factor of 4 reduction in die size through all 4 generations. The first 3 reductions were due to improved circuit architectures as well as MEMS designs using the subsequent generations of $i$ MEMS. The ADXL50 was chosen to be a force-feedback design because the stability of the polysilicon was not well established.
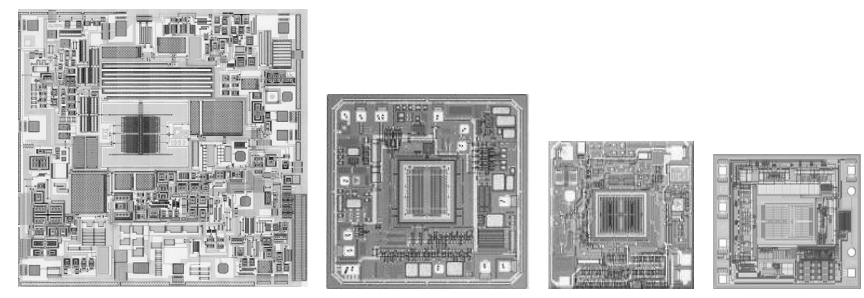

\begin{tabular}{|c|c|c|c|c|c|}
\hline & $\begin{array}{c}\text { ADXL50 } \\
(1994)\end{array}$ & $\begin{array}{c}\text { ADXL76 } \\
(1996)\end{array}$ & $\begin{array}{c}\text { ADXL78 } \\
(2001)\end{array}$ & $\begin{array}{c}\text { ADXL40 } \\
(2004)\end{array}$ & \\
\hline Die Area & 10.8 & 5.4 & 2.7 & 2.5 & $\mathrm{~mm}^{2}$ \\
\hline MEMS Area & 0.43 & 0.38 & 0.27 & 0.22 & $\mathrm{~mm}^{2}$ \\
\hline$\%$ MEMS & $4.0 \%$ & $7.0 \%$ & $10 \%$ & $8.8 \%$ & \\
\hline Cs & 100 & 100 & 40 & 160 & $\mathrm{fF}$ \\
\hline fo & 25.0 & 24.5 & 24.5 & 12.5 & $\mathrm{kHz}$ \\
\hline Noise & 6.0 & 1.0 & 1.0 & 1.0 & $\begin{array}{c}\mathrm{mgee} \\
\text { rt.hz }\end{array}$ \\
\hline Offset & 3.0 & 1.0 & 0.5 & 0.5 & gee \\
\hline
\end{tabular}

Figure 5: The airbag crash sensor family of ADI - from the original ADXL50 (1991) to the $4^{\text {th }}$-generation accelerometer using the new SOIMEMS process technology.

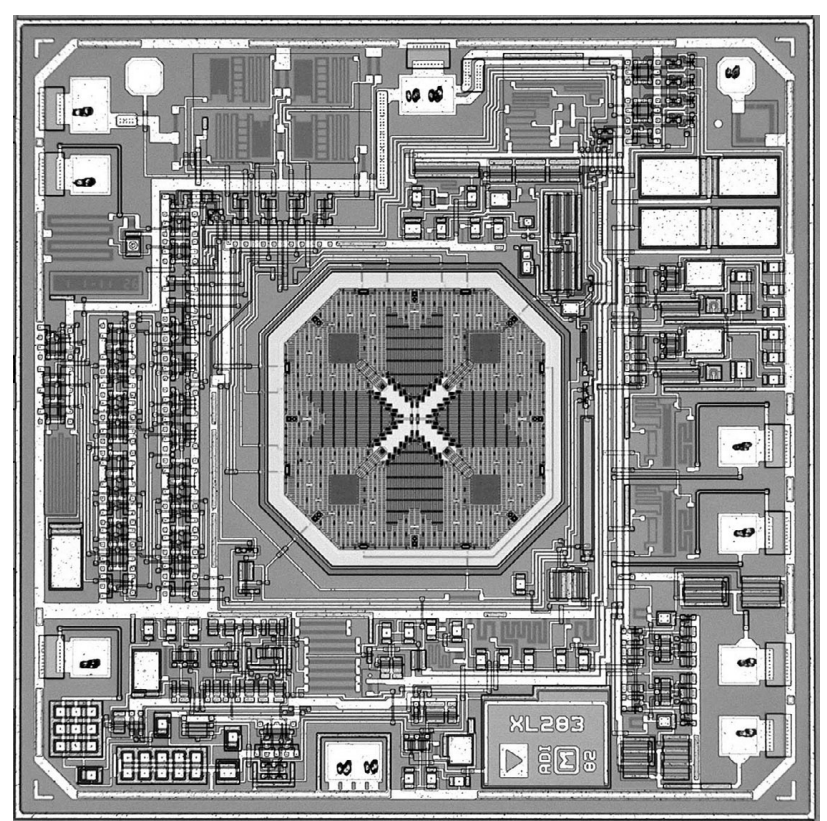

Figure 6: ADXL203: a 2-axis accelerometer with \pm 50 mgee null bias stability and $110 \mu \mathrm{gee} / \mathrm{rt}$.hz noise.

After proving the stability of polysilicon as a structural material, an open-loop architecture was implemented in the ADXL76, which provided a factor of two decrease in die size.

The next factor of 2 decrease in die size with the ADXL78 also produced a more robust sensor to overload accelerations. This had become a requirement when the accelerometers were placed in the periphery of the automobile, which is exposed to much higher accelerations during impacts (i.e., side impacts). This device uses a differential circuit and MEMS architecture and feedback (i.e., not force feedback) to help reduce any undesirable overload response.

The most recent step in airbag crash sensors is the ADXL40, which uses the SOIMEMS technology with post-package trim. The scale factor and bandwidth of the part can be electrically selected by the user. Like the MEMS-only sensors that have similar electrostatic gap aspect ratios, the ADXL40 uses squeeze film damping to preserve velocity during an overload event.

In addition to the reduction in die size, improving key performance specifications has also been a goal. A good example of how the performance of a MEMS device has been improved is the ADXL203, a second-generation dual-axis accelerometer with \pm 1.7 gee full-scale dynamic range (Figure 6). The first dual-axis accelerometer, the ADXL202, had a null accuracy of 500 mgee which made DC measurement of tilt difficult. With the advent of VDC systems in cars, it was recognized that there was a clear market for a DC capable dual axis accelerometer. Since postpackage trimming was not available at the time, the designers needed to produce a device that would be inherently insensitive to packaging and thermal stresses. It was recognized early that by changing the mounting of the spring-mass and differential capacitor finger system from perimeter to die center that the magnitude of the die distortion that causes the offset shifts would be significantly reduced. Through experimental test chips and extensive modeling, the optimal position of anchors was obtained. The resulting ADXL203 improved the null accuracy to a typical value of 25 mgee and a total of \pm 50 mgee over the operating 
temperature $\left(-40^{\circ} \mathrm{C}\right.$ to $\left.125^{\circ} \mathrm{C}\right)$. The noise of the accelerometer was also reduced to $110 \mu \mathrm{gee} / \mathrm{rt}$.hz by improving the beam biasing architecture. These performance improvements allowed the product to measure absolute tilt to 3 degrees and resolve tilt differences of less than 0.01 degrees.

The most challenging device by far to develop has been the ADXRS150, a vibratory rate gyro (Figure 7) $[8,9]$. The two uncoupled structures are oscillated in anti-phase and the resulting coriolis motion is synchronously detected by the integrated accelerometer. The minimum detectable coriolis displacement is $1.6 \times 10^{-4} \AA$ or about 2 orders of magnitude lower than the ADXL203. This level of performance requires the structures to be manufactured with improved precision in order to prevent structure imperfections from producing feedthrough of resonator motions into the coriolis signal. The development of the gyro is a great example of the strong design and manufacturing interaction that is required by all MEMS products. During the development of this device the designers requested that the process engineers guarantee the CD matching within a gyro structure be $20 \mathrm{~nm}$. Initially, this level of matching was viewed as a show stopper for a high-yield gyro. However, through careful data collection, experimentation, and design improvements the manufacturing requirements of the gyro were met successfully.

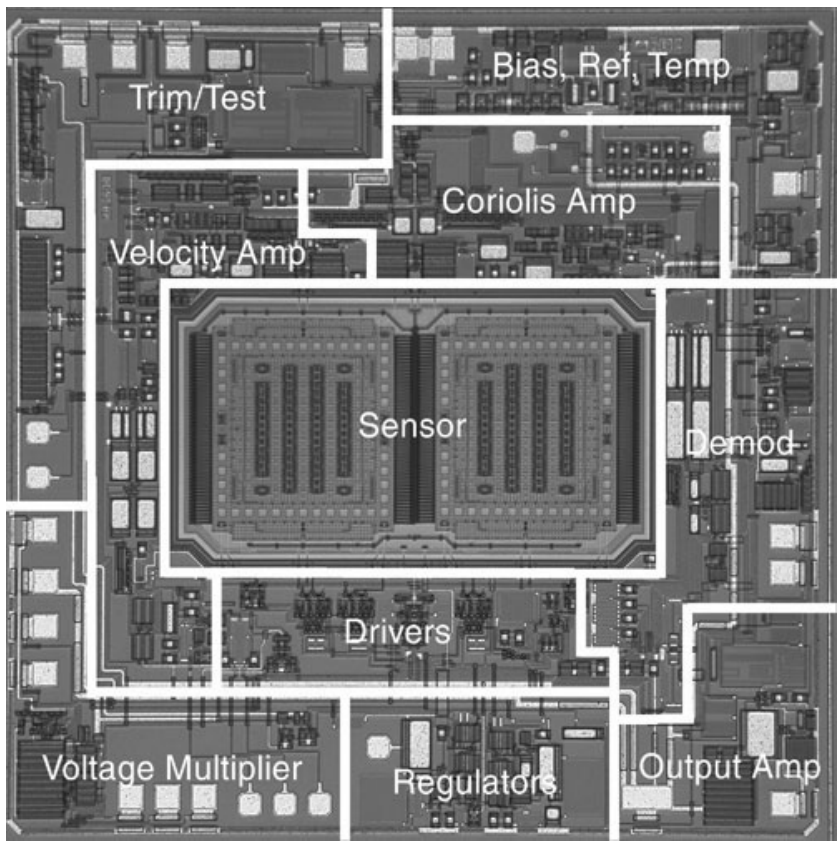

Figure 7: ADXRS150: a z-axis gyroscope with $0.05 \mathrm{deg} / \mathrm{rt.sec}$ noise integrated on a $3 \times 3 \mathrm{~mm}$ die.

\section{DESIGN-MANUFACTURING INTERACTION}

As stated earlier, MEMS products require a very tight coupling between design/product development and manufacturing. Around each product at ADI is a cross-functional team of design and manufacturing people. This highly integrated productdevelopment method is in stark contrast with what typically transpires with most integrated-circuit products, especially purely digital circuits. Here the designer works in isolation of the circuit technology, relying solely on the design and layout specification to guide their design. Even though integrated MEMS technology has become a mature process, it is these close working relationships that have enabled the successful release and enhancement of all of the integrated inertial MEMS products at ADI.

There are of course layout and design specifications that are provided to the MEMS designers. Manufacturing tolerances are also provided, such as the mean and standard deviation of all the critical MEMS geometry and material properties. Some of these parameters are a result of in-line monitoring of product wafers, while others are tracked or characterized on a more infrequent basis. The incorporation of these tolerances into the design is vital to producing high-yield products. Use of statistical-processing controls, such as $\mathrm{C}_{\mathrm{pk}}$ monitoring, can be use to monitor and control the health of the manufacturing line. The close working relationship between design and manufacturing has produced MEMS products with yields the same as integrated circuits products without MEMS as well as a profitable business.

\section{FUTURE TECHNOLGY \& PRODUCTS}

The next generation of products to address the inertial automotive and consumer electronics markets will continue down the paths of higher efficiency/utilization, increased use of standard IC equipment, and further product differentiation. As described throughout this paper, the die sizes will continue to drop. A $1-\mathrm{mm}^{2}$ die accelerometer is within reach with the integrated approach, which will drop the die cost by another factor of 2.5 . It is unlikely that the two-chip approaches will reach even $3-\mathrm{mm}^{2}$ due to the large sensors required by the extra parasitic capacitances. A successful in-situ capping technology might be released to manufacturing, which would reduce the die sizes even further.

Most inertial-sensor manufacturers are working on or have released a LFCSP-style package. The next stages will reduce the size and thickness of these packages. The thickness, surprisingly, is the more stringent requirement which should approach the industry standard of $0.9 \mathrm{~mm}$. Eventually, the wafer-scale packaging (WSP) trend in integrated circuits will be adopted by MEMS companies. In this package the plastic package is completely removed and the silicon die is flip-chip mounted directly to the board.

Testing of inertial devices will also be an area of very active development and should evolve to the point where the application of an acceleration or angular rate will not be required. Instead, an electrical stimulus will be adequate to insure the device has met the required performance tolerance. With the evolution to smaller and lighter packages, such as WSP, there will be many more challenges with simply handling the packages and making reliable contacts. Part of this progress will be the increased understanding of the customers to the real requirements for their applications. For example, does a product need to have DC response when relative responses are all that are required? As a result, sensorspecification requirements might relax if lower prices can be obtained.

The evolving capping and packaging technologies and integration techniques will lead to other product developments beyond inertial sensors. The core technology of surface micromachining using polysilicon and SOI has already demonstrated a great variety of devices, such as oscillators, variable capacitors, pressure sensors, and microphones. However, the cost of these devices has been prohibitive for the development of most of their corresponding high-volume markets. The cost structures and technology evolution of inertial MEMS will 
eventually allow access to these other high-volume non-inertial markets.

\section{SUMMARY}

Inertial MEMS products are commodities. The selection of a part is made almost solely on the unit price because there are at least two vendors who provide similar performance, quality, and reliability for most high-volume products. To continue to be competitive both in the existing markets and emerging markets, a highly active technology development is ongoing throughout the entire industry. Even though ADI produces $10^{6}$ MEMS devices/week it is nowhere near enough to independently drive to the lowest cost on a custom process. To meet customer expectations, MEMS manufacturers must adopt existing capacities and capabilities anywhere possible for fabrication, assembly, and test. Perhaps in the not-too-distant future, when accelerometers reach $\$ 1 /$ axis or less, will the total market revenue be great enough to begin to drive the costs down. Until that time, we can look forward to many more evolutions of inertial MEMS products and likely a few new applications that leverage this technology base.

\section{ACKNOWLEDGEMENTS}

Thanks go to everyone who has contributed to the success of ADI's inertial MEMS technology. This paper presents the accumulated accomplishments and hard work by everyone within the Micromachined Products Division. The author would also like to thank the MPD management team for supporting the publication of the overview of ADI's technology evolution.

\section{REFERENCES}

1. S. C. Terry, "A Miniature Silicon Accelerometer with BuiltIn Damping," Solid-State Sensor and Actuator Workshop, Hilton Head Island, S.C., June 1988, pp. 114-116.

2. P. W. Barth, F. Pourahmadi, R. Mayer, J. Poydock, and K. Peterson, "A Monolithic Silicon Accelerometer with Integral Air Damping and Overrange Protection," Solid-State Sensor and Actuator Workshop, Hilton Head Island, S.C., June 1988, pp. 35-38.

3. K. E. Peterson, "Bringing MEMS to Market," Solid-State Sensor and Actuator Workshop, Hilton Head Island, S.C., June 2000, pp. 60-64.

4. T. A. Core, W. K. Tsang and S. J. Sherman, "Fabrication Technology for an Integrated Surface Micromachined Sensor," Solid State Technology, Oct 1993, pp. 47-53.

5. K. H.-L. Chau, R. E. Sulouff, Jr. "Technology for the highvolume manufacturing of integrated surface-micromachined accelerometer products," Microelectronics Journal, vol. 29, 1998, pp. 579-586.

6. C. M. Roberts, Jr., L. H. Long, P. A. Ruggerio, "Method for Separating Circuit Dies from a Wafer," U.S. Patent 5,362,681, 1994.

7. L. E. Felton, M. Duffy, N. Hablutzel, P. W. Farrell, and W. A. Webster, "Low-Cost Packaging of Inertial MEMS Devices," Proceedings of $36^{\text {th }}$ International Symposium on Microelectronics, Boston, MA, Nov. 2003, pp. 402-406.

8. J. A. Geen, "A Path to Low Cost Gyroscopy," Solid-State Sensor and Actuator Workshop, Hilton Head Island, S.C., June 1998, pp. 51-54.

9. J. A. Geen, S. J. Sherman, J. F. Chang, S. R. Lewis, "SingleChip Surface Micromachined Integrated Gyroscope With $50^{\circ} / \mathrm{hr}$ Allan Deviation," Journal of Solid-State Circuits, vol. 37 , no. 12 , pp. $1860-1866$. 